\title{
Galaxy properties and photometric redshifts
}

\author{
Uta Fritze - v. Alvensleben ${ }^{1}$, Jens Bicker ${ }^{1}$ and Barbara Cunow ${ }^{2}$ \\ ${ }^{1}$ Universität Göttingen, Germany, ${ }^{2}$ Unisa, Pretoria, South Africa \\ email: ufritze@astro.physik.uni-goettingen.de
}

Keywords. Galaxies: evolution, high-redshift, starbursts, interactions

We present GALEV evolutionary synthesis models for Simple Stellar Populations (SSPs = single burst, single metallicity) like star clusters or galaxy pixels and for galaxies of various types on the basis of their respective typical star formation histories, ranging from exponentially declining on a timescale of $1 \mathrm{Gyr}$ for the classical elliptical model through constant for Sd galaxies, and also allowing for starbursts of various strengths occurring at various evolutionary stages. Models yield the time evolution of the stellar population in terms of color-magnitude diagrams CMDs $(U \ldots K)$, spectra $(90 \AA \ldots 160 \mu \mathrm{m})$ including gaseous emission in terms of lines and continuum, luminosities, colors and M/L-ratios in various filter systems (e.g. Johnson $U \ldots K$, HST, Washington, Strömgren), and Lick indices (http://www.astro.physik.uni-goettingen.de/ ${ }^{\sim}$ galev). For galaxies, the redshift evolution is obtained from the time-evolving spectra assuming a standard cosmology $\left(\mathrm{H}_{0}=65, \Omega_{0}=0.3, \Omega_{\Lambda}=0.7\right)$ and consistently accounting for evolutionary and cosmological corrections as well as for the attenuation of light from distant galaxies by intervening HI.

Folding over the spectra a set of filters (e.g. Johnson $U \ldots K$ ) yields Spectral Energy Distributions (SEDs), i.e. sets of luminosities in these filters. For star clusters and pixel-by-pixel analyses, we have calculated a grid of 120000 SSP models for 5 metallicities $-1.7 \leqslant[\mathrm{Fe} / \mathrm{H}] \leqslant+0.4,1200$ ages $4 \mathrm{Myr} . .14 \mathrm{Gyr}$, and 20 extinction values $0 \leqslant E(B-V) \leqslant 1$ with a starburst extinction law. For SSPs the SEDs scale with mass. For galaxies we have calculated a large grid of dustfree model SEDs for E, S0, Sa, Sb, Sc, Sd, undisturbed and with starbursts of various strengths $10 \%, 30 \%, 50 \%, 70 \%$ starting at various redshifts, in their redshift evolution from $z=6$ to $z=0$ (Bicker \& Fritze - v. Alvensleben 2006). B-band luminosities of undisturbed galaxies are gauged to match the average observed ones at $z=0$. Undisturbed galaxies are modelled in a chemically consistent way, i.e. accounting for the increasing initial metallicities of successive stellar generations (cf. Bicker et al. 2004). For SSPs (star clusters and galaxy pixels) we developed an SED analysis tool AnalySED (Anders et al. 2004) that compares an observed SED obtained from multi-band imaging to the grid of model SEDs and, by means of a $\chi^{2}-$ algorithm, returns the age, metallicity, $\mathrm{E}(B-V)$, and mass including their respective $\pm 1 \sigma$ uncertainties. In Bicker \& Fritze - v. Alvensleben (in prep.) we extended this tool to analyse multi-band galaxy SEDs in terms of galaxy types, redshifts, eventually burst strengths and ages, etc. Analysing artificial star clusters and galaxies we find that accurate (i.e. to $\leqslant 0.1 \mathrm{mag}$ ) photometry in at least 4 wavelengths bands covering as long a wavelength basis as possible $(U \ldots K)$ allows to very precisely recover the input parameters (Anders et al. 2004, de Grijs et al. 2005). The $U$-band is of crucial importance for ages, metallicities (and extinctions) of young stellar populations, the NIR is important for metallicities and mass contributions of older stellar populations and for galaxies at higher redshift. SALT's unique $U$-band sensitivity and its NIR arm together with the large collecting area and the large field of view make it the ideal instrument for this kind of multi-band imaging studies.

The comparison of our results for the HDF galaxies (WFPC2 F300W...F814W, NICMOS F110W \& F160W) showed that our photometric redshifts agree with the spectroscopic ones to better than $5-10 \%$. Models also show that the use of intermediate band filters (e.g. Stroemgren + extension to longer wavelength) will allow for even higher precision in photometric redshifts for galaxies to $z<2$. Our analysis showed that all galaxies in the HDF are well described by undisturbed chemically consistent models, starbursts or post-starbursts, that there are no bright 
ellipticals at $z>1$, but many bright blue galaxies with strong starbursts at $4>z>0.5$, many extremely red post-starburst galaxies at $2.5>z>1$, the now dust-free successors of very strong bursts at $4>z>2$ (Bicker \& Fritze - v. Alvensleben 2006).

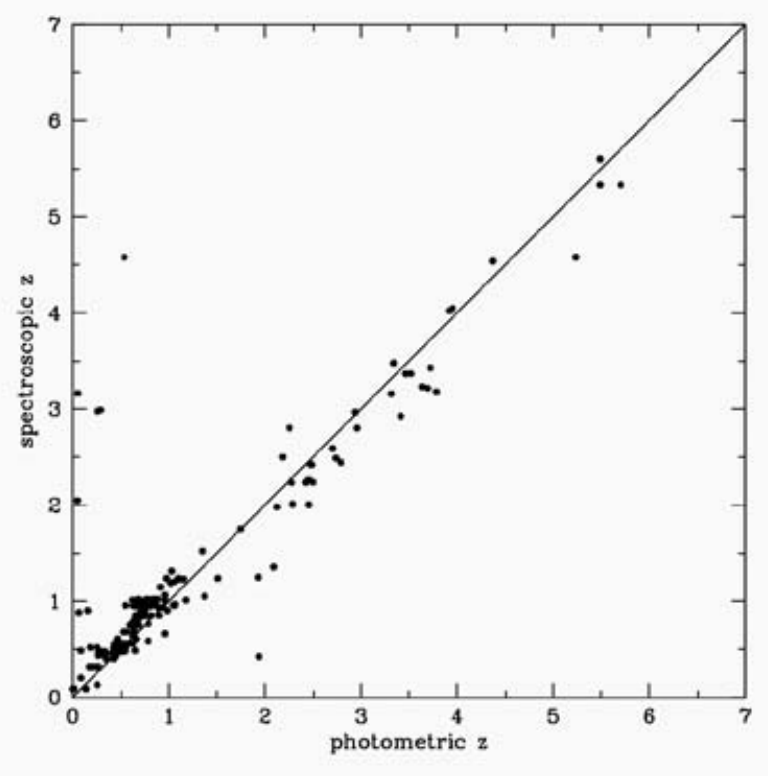

Figure 1. Comparison of our photometric redshifts for HDF galaxies with the spectroscopic redshifts available for a subsample (Bicker \& Fritze - v. Alvensleben, in prep.).

Pixel-by-pixel analyses of HST ACS BVI data for the interacting starburst galaxy Tadpole revealed very young stars and star clusters not only in distinct regions of the main body but also all along the $180 \mathrm{kpc}$ long tidal tail and showed star cluster formation to be a major mode of star formation ( $>35 \%$ in mass) with $\sim 70 \%$ of the $B$-light (and $\sim 40 \%$ in $I$ ) coming from young star clusters as opposed to field stars (de Grijs et al. 2003).

Multi-band photometry and its analysis can very economically been done for all objects in a field and reaches much deeper than spectroscopic studies, yet it allows to recover the properties of the stellar population to high accuracy. In particular, at typical photometric accuracies broad band photometry with useful passband combinations is as powerful in disentangling ages, metallicities and dust of SSPs and in revealing star formation histories and photometric redshifts of galaxies as is spectroscopy at typical $\mathrm{S} / \mathrm{N}$.

\section{Acknowledgements}

UFvA gratefully acknowledges travel support from the Deutsche Forschungsgemeinschaft under Fr 916/15-1. This work was partly funded by the Deutsche Forschungsgemeinschaft under Fr 916/10-1-2 and Fr 916/11-1-2.

\section{References}

Anders, P., Bissantz, N., Fritze - v. Alvensleben, U. \& de Grijs, R. 2004, MNRAS 347, 196

Bicker, J., Fritze - v. Alvensleben, U., Moeller, C.S. \& Fricke, K.J. 2004, A\&\&A 413, 37

Bicker, J. \& Fritze - v. Alvensleben, U. 2006, A\& A, in press

de Grijs, R., Lee, J., Mora Herrera, C. \& Fritze - v. Alvensleben, U. 2003, New Astron 8, 155

de Grijs, R., Anders, P., Lamers, H.J.G.L.M., Bastian, N., Fritze - v. Alvensleben, U., Parmentier, G., Sharina, M.E. \& Yi, S. 2005, MNRAS 359, 874 